\title{
Comparison of numerical solution strategies for population balance model of continuous cone mill
}

\author{
Satyajeet S. Bhonsale ${ }^{\mathrm{a}, \mathrm{b}}$, Dries Telen ${ }^{\mathrm{a}}$, Bard Stokbroekx ${ }^{\mathrm{b}}$, Jan Van Impe ${ }^{\mathrm{a}, *}$

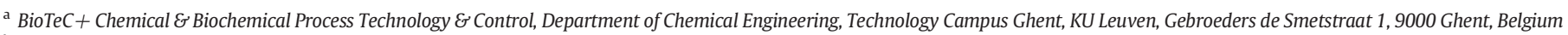 \\ ${ }^{\mathrm{b}}$ Crystallisation Technology Unit, Pharmaceutical Development and Manufacturing Sciences, Janssen Pharmaceutica, Turnhoutseweeg 30,2340 Beerse, Belgium
}

\section{A R T I C L E I N F O}

\section{Article history:}

Received 30 May 2018

Received in revised form 8 January 2019

Accepted 18 January 2019

Available online 24 January 2019

\section{Keywords:}

Population balance model

Cone mills

Pharmaceutical development

Quality by design

\begin{abstract}
A B S T R A C T
Population balance models are routinely used for modeling particulate processes involving breakage, agglomeration, crystallisation, etc. In this study, a comparison between three different numerical solution strategies for breakage population balance models is presented. Results are obtained for the fixed pivot technique, moving pivot technique and the cell average technique. These techniques are first compared for models whose solutions are analytically available. The comparison shows that fixed pivot lacks the accuracy of both moving pivot and cell average at coarser grids. At finer grids, all the three solution strategies give similar errors. With increasing grid fineness, all the three methods converge to a grid independent solution. Moving pivot is computationally the most expensive methods, followed by the cell average and fixed pivot. These methods are then applied to a conical screen mill modelled by a continuous population balance equation. For all the discretizations considered, cell average computes a higher median particle size value than the fixed pivot and moving pivot.
\end{abstract}

C 2019 Published by Elsevier B.V.

\section{Introduction}

Milling or particle size reduction is an important processing step in the manufacturing chain of the pharmaceutical industry. Milling is used to delump blended active pharmaceutical ingredients (APIs), reduce particle size of oversized granules or to break compacted ribbons. The particle size distribution (PSD) of the granules is an important critical quality attribute that influences the quality of the final product, e.g., extremely fine granules tend to have poor flowability, while large granules have a detrimental effect on tablet strengths. The PSD of the seed material to a crystallizer affects the morphology and size control of the crystals. Even prior to the drug product manufacturing stage, milling remains an important step, e.g., the PSD plays an important role to improve the dissolution rate of the Biopharmaceutics Classification System II drugs $[1,2]$.

The cone mill is a popular size reduction equipment used regularly in the pharmaceutical industry for a variety of reasons. It has been used for delumping blended APIs, wet and dry granulated materials, and to break tablets for API recovery. The cone mill consists of a rotating impeller which causes impacts between the particles and the impeller. The

Abbreviations: API, Active Pharmaceutical Ingredient; PSD, Particle Size Distribution; PBM, Population Balance Models; FPT, Fixed Pivot Technique; CAT, Cell Average Technique; MPT, Moving Pivot Technique.

* Corresponding author.

E-mail addresses: satyajeetsheetal.bhonsale@kuleuven.be (S.S. Bhonsale), dries.telen@kuleuven.be (D. Telen), bstokbro@its.jnj.com (B. Stokbroekx), jan.vanimpe@kuleuven.be (J. Van Impe). particles stay in the mill until they are reduced to a size smaller than the screen aperture. Different impeller shapes are available along with a variety of screens with different shapes and apertures. The screen size is the most significant parameter affecting the particle size of the milled product $[3,4]$. Even then, the impeller shape and speed, along with the screen size have a statistically significant impact on the final size distribution [5]. For the same impeller shape, either an increase in impeller speed or a decrease in screen size leads to a lower mean particle size. The effect of different screen shapes was investigated by Vanarase et al. [6] who report that the grated type screens show greater milling capacity as opposed to rounded type screens. The primary mode of breakage in dry cone milling is impact attrition. Although the screen influences the final PSD, it does not influence the mode of breakage [7]. Barrasso et al. [8] studied the cone mill operating under a continuous starve feed mode, which is the standard mode of operation in continuous or semi-continuous manufacturing. They reported that the API density, the screen size and the impeller speed are the parameters which affect the residence time. These parameters can be used as design parameters to represent the degree of breakage.

Despite the importance of milling in the solid dosage manufacturing, the mechanistic understanding of the process remains poor. This is primarily due to the lack of first principle models describing particle breakage. However, the Quality by Design initiative pushes for the development of mechanistic or semi-mechanistic predictive models that can enhance the black-box nature of the process and contribute to its better understanding. To this extent, various approaches have been utilised to describe comminution processes. Fracture mechanics 
based models utilize the Hertzian theory of normal stresses and flaw analysis to describe particle breakage [9]. The fracture mechanics based attrition model has been used to estimate volumetric wear of granules in cone mills, and to conclude that attrition indeed is the primary breakage mode [7]. Discrete element models (DEM) use numerical schemes to solve Newton's equations of motion and contact mechanics to predict particle trajectories [10]. This leads to a highly mechanistic model and has been applied to a vast range of pharmaceutical operations [11-13]. DEMs have been used to simulate breakage processes like ball mills, spiral jet mills, and impact mills [14-17]. However, due to the time-varying nature of the PSD, the application of DEMs as predictive models remains rather limited [18]. Furthermore, the contact mechanics based algorithms in DEMs lead to extremely small computational steps making the DEMs run times extensive. For a realistic scenario the number of particles within a system is often in the range of $10^{9}$ particles, leading to even more computationally intensive simulations. More recently, multiscale methods involving DEMs coupled with population balances have been developed for many cases. Capece et al. [19] build upon the mastercurve of Vogel and Peukert [20] to determine a mechanistic breakage constant using particle interactions simulated from DEM. Furthermore, in [21] an approach to couple DEM and population balance is presented. Such a coupling approach has been applied to model the cone mill [22], impact mill [17,23], etc.

The focus of this work lies on pure breakage population balance models. Population balance models have been successfully used in modeling particulate processes like granulation, dissolution, crystallisation, mixing, etc. A large body of literature considers application of PBMs for breakage processes as well. For processes involving pure breakage, and under the assumption of well-mixedness, a 1-dimensional batch PBM in terms of particle size can be formulated as:

$\frac{\partial n(t, x)}{\partial t}=\underbrace{\int_{x}^{\infty} b(x, y) S(y) n(t, y) d y}_{\mathscr{B}(t, x)}-\underbrace{S(x) n(t, x)}_{\mathcal{D}(t, x)}$

The term $\mathscr{B}(t, x)$ on the right-hand side of the equation represents the birth of particle by the breakage process. The breakage function $b$ $(x, y)$ is the probability function describing the formation of particles of size $x$ by the breakage of the particles of size $y$. The selection function $S(x)$ describes the rate of breakage of particles of size $x$. The term $\mathcal{D}(t, x)$ on the right describes the death of the particle because of breakage. In case particle size is represented by the particle volume in the 1D PBM, the breakage function should satisfy the following consistency condition.

$\int_{0}^{y} x b(x, y) d x=y$

This property states that the total volume of particles formed due to the breakage of a particle is equal to the volume of the original particle itself.

Given the complex integro-differential nature of the equations, analytical solution of the PBM is available only for a few simplified cases. Most often, numerical techniques are required to solve the PBM. Several numerical schemes have been proposed to solve the PBM. These methods include, among others, the method of successive approximations [24], the method of moments [25-28], Monte Carlo methods $[29,30]$, sectional methods, etc. Ramkrishna [24] provides the details of all these methods along with the theory behind general population balances.

Sectional methods divide the continuous size distribution into discrete bins by discretizing the continuous property vector (in case of this 1D PBM, the particle size). The partial integro-differential equation is then converted into a system of ordinary differential equations (ODEs) describing the time evolution of the particle number in each bin. Sectional methods focus on predicting certain selected properties of the system like total particle number and total mass. These methods are highly popular in engineering applications because of their simplicity and low computational costs. A variety of sectional methods have been proposed in the literature [31-38].

The objective of this paper is to compare the numerical results obtained by three different sectional approaches applied to an engineering system described by a population balance model. The fixed pivot [34], the moving pivot [35], and the cell average [37] are considered for this comparison. Comparison of solution strategies has been the subject of some studies [e.g., 39,40]. However, these compare only simple generic case studies which are analytically solvable. In this paper, the three methods are compared for a practical case study along with the analytical cases. This case refers to the cone mill described previously. In this study, the model developed by Barasso et al. [8] is considered.

The article is organised as follows. First, the three numerical techniques to solve the PBM are described along with the cone mill model which will be used. Next, the three techniques are numerically compared for various case studies for which analytical solutions are available and for the cone mill model.

\section{Theoretical and numerical methods}

The first subsection describes the three numerical schemes which will be used to solve the PBMs in this paper. The case studies with analytical solutions are introduced in the second subsection, whereas the cone mill model developed by Barrasso et al. [8] is described in the third subsection.

\subsection{Numerical techniques for solution of PBMs}

\subsubsection{Fixed pivot technique}

The fixed pivot technique (FPT) proposed by Kumar and Ramkrishna [34] is based on birth modification. The continuous size domain is first discretized into $I$ cells of size $\Delta x_{i}=x_{i-1 / 2}-x_{i+1 / 2}, i=1, \ldots, I$. Every individual cell $\left[x_{i-1 / 2}, x_{i+1 / 2}\right]$ is represented by a size $x_{i}$ called the pivot. The particle distributions are considered to be point masses at these pivot points. Thus, the entire size distribution can be represented by

$N_{i}(t)=\int_{x_{i-1 / 2}}^{x_{i+1 / 2}} n(x, t) d x, \quad \forall i=1, \ldots, I$

\section{Table 1}

Simulation conditions and computational times (in seconds) for the case studies with analytical solution.

\begin{tabular}{|c|c|c|c|c|c|c|c|}
\hline \multirow[t]{2}{*}{ Case } & \multirow[t]{2}{*}{$x_{\min }$} & \multirow[t]{2}{*}{$x_{\max }$} & \multirow[t]{2}{*}{$T_{\text {end }}$} & \multirow{2}{*}{$\begin{array}{l}\text { Grid } \\
\text { density }\end{array}$} & \multicolumn{3}{|c|}{ Computational time in seconds } \\
\hline & & & & & FPT & MPT & CAT \\
\hline \multirow[t]{5}{*}{1} & \multirow[t]{5}{*}{$10^{-9}$} & \multirow[t]{5}{*}{1} & \multirow[t]{5}{*}{1000} & 15 & 0.22 & 71.61 & 0.36 \\
\hline & & & & 30 & 0.68 & 693.27 & 0.77 \\
\hline & & & & 90 & 5.49 & $5.86 \times 10^{3}$ & 5.55 \\
\hline & & & & 150 & 14.2 & $1.84 \times 10^{4}$ & 14.5 \\
\hline & & & & 200 & 25.65 & $4.23 \times 10^{4}$ & 27.48 \\
\hline \multirow[t]{5}{*}{2} & \multirow[t]{5}{*}{$10^{-9}$} & \multirow[t]{5}{*}{1} & \multirow[t]{5}{*}{1000} & 15 & 0.25 & 60.31 & 0.41 \\
\hline & & & & 30 & 0.72 & 727.66 & 1.17 \\
\hline & & & & 90 & 5.7 & $1.34 \times 10^{4}$ & 13.26 \\
\hline & & & & 150 & 16.94 & $3.68 \times 10^{4}$ & 21.19 \\
\hline & & & & 200 & 25.77 & $5.77 \times 10^{4}$ & 32.47 \\
\hline \multirow[t]{5}{*}{3} & \multirow[t]{5}{*}{$10^{-9}$} & \multirow[t]{5}{*}{125} & \multirow[t]{5}{*}{10} & 15 & 0.11 & 55.78 & 0.17 \\
\hline & & & & 30 & 0.29 & 299.78 & 0.68 \\
\hline & & & & 90 & 3.56 & $5.4 \times 10^{3}$ & 5.24 \\
\hline & & & & 150 & 8.17 & $2.30 \times 10^{4}$ & 13.62 \\
\hline & & & & 200 & 15.23 & $4.38 \times 10^{4}$ & 23.9 \\
\hline \multirow[t]{5}{*}{4} & \multirow[t]{5}{*}{$10^{-9}$} & \multirow[t]{5}{*}{125} & \multirow[t]{5}{*}{5} & 15 & 0.496 & $1.28 \times 10^{3}$ & 3.22 \\
\hline & & & & 30 & 1.28 & $1.44 \times 10^{4}$ & 10.87 \\
\hline & & & & 90 & 10.07 & $3.6 \times 10^{5}$ & 129.67 \\
\hline & & & & 150 & 28.62 & - & 415.08 \\
\hline & & & & 200 & 15.30 & - & 726.22 \\
\hline
\end{tabular}


The general idea behind the FPT is to redistribute the particles formed in a size class into neighbouring classes. If a new particle is born at a size other than that of the pivot, it is divided between the neighbouring pivots such that any two integral properties are conserved. The equation for the FPT by conserving the number and mass are given as follows

$\frac{d N_{i}}{d t}=\sum_{j \geq i}^{I} \eta_{i, j} S_{j} N_{j}-S_{i} N_{i}$

where,

$\eta_{i, j}=\int_{x_{i}}^{x_{i+1}} \frac{x_{i+1}-x}{x_{i+1}-x_{i}} b\left(x, x_{j}\right) d x+\int_{x_{i-1}}^{x} \frac{x-x_{i+1}}{x_{i}-x_{i-1}} b\left(x, x_{j}\right) d x$

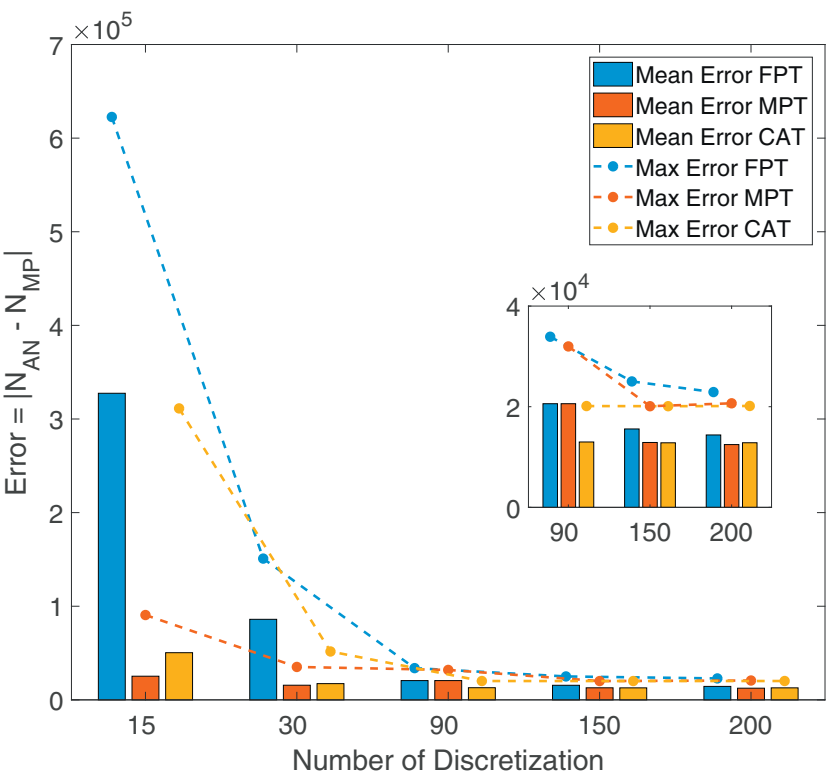

(a) Case 1

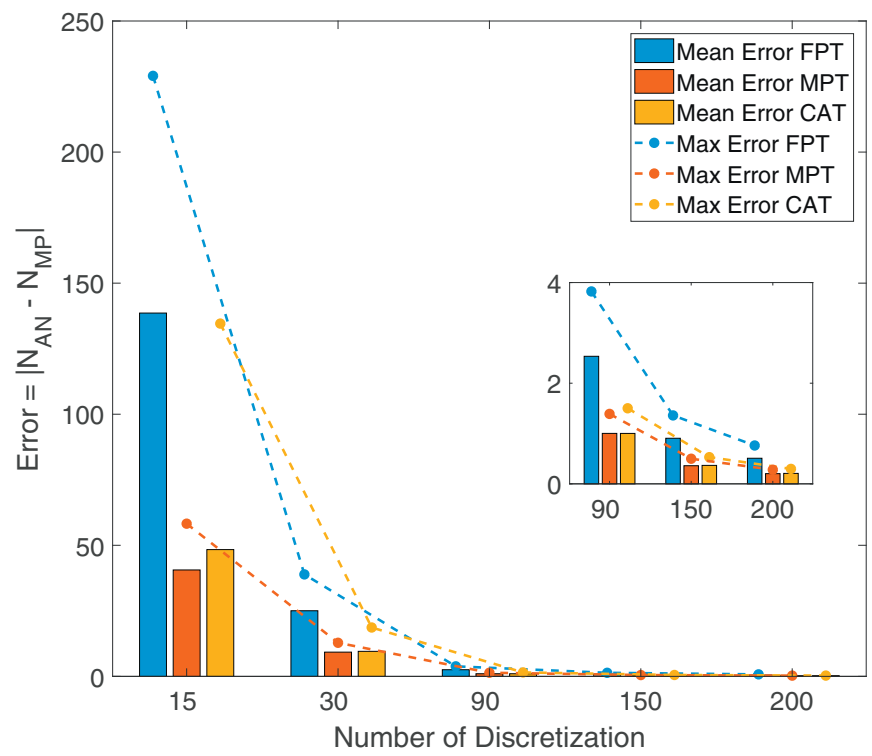

(c) Case 3
However, the main disadvantage of FPT is that it overpredicts the number density in the finer size range for coarse grids. To overcome this issue, the authors proposed the moving pivot technique which is described next.

\subsubsection{Moving pivot technique}

The moving pivot technique (MPT) aims to address the issue of overprediction by allowing a varying pivot location. This allows to account for the non-uniformity of the size distribution in a size range. The equations for MPT conserving the first two moments: the total number and total mass are

$\frac{d N_{i}}{d t}=\sum_{j \geq i}^{I} \beta_{i, j}^{(I)} S_{j} N_{j}-S_{i} N_{i}$

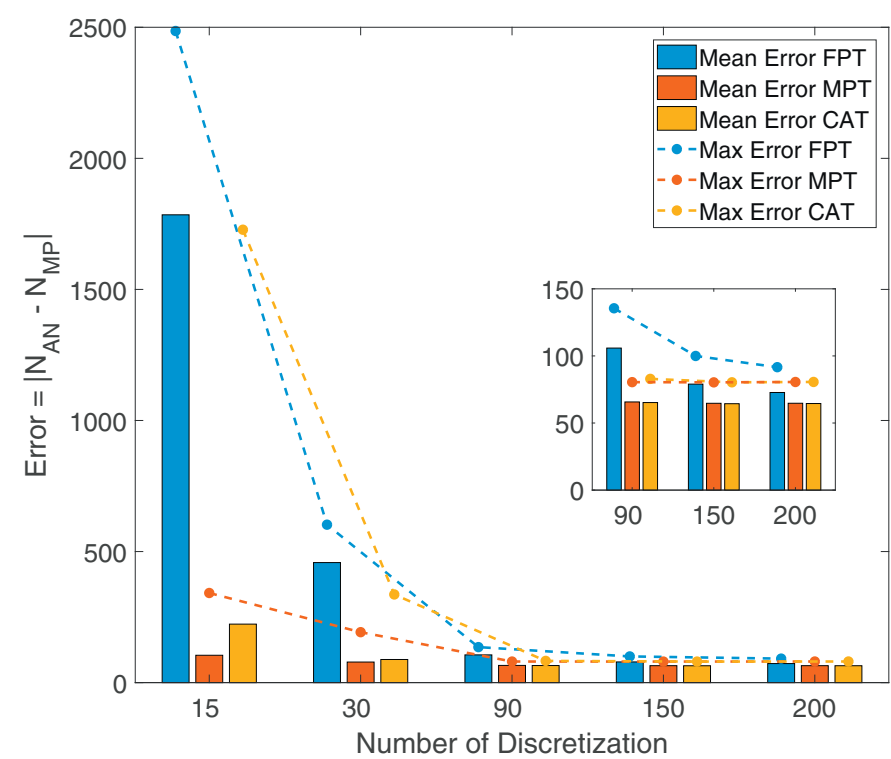

(b) Case 2

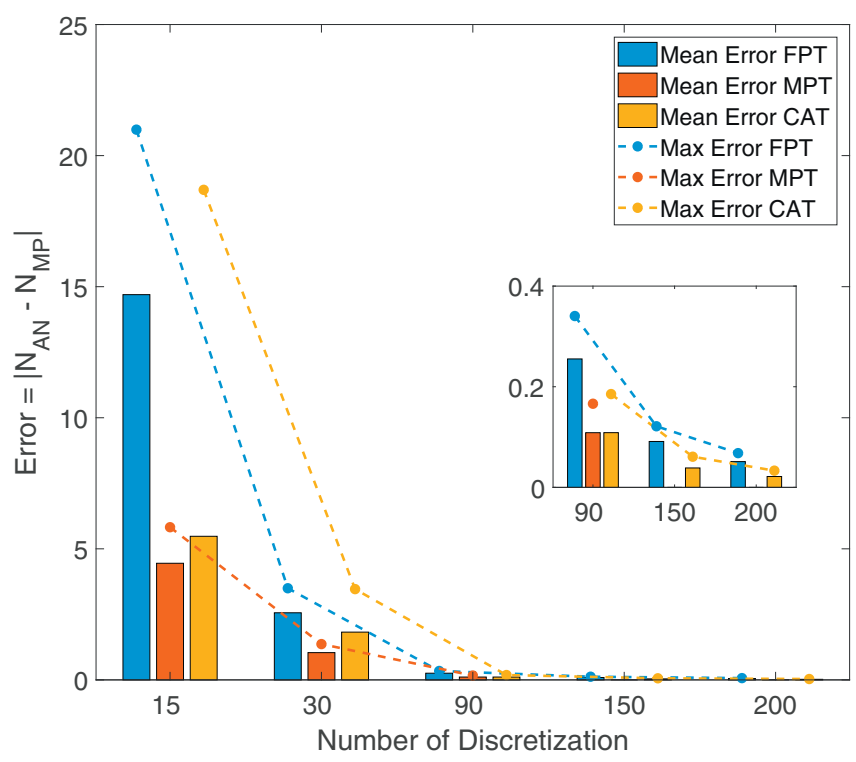

(d) Case 4

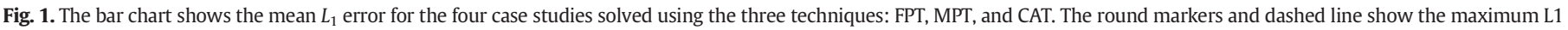

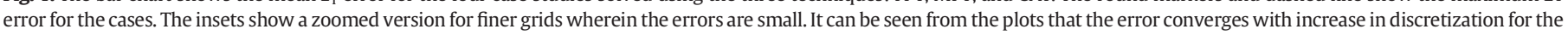
three methods. 


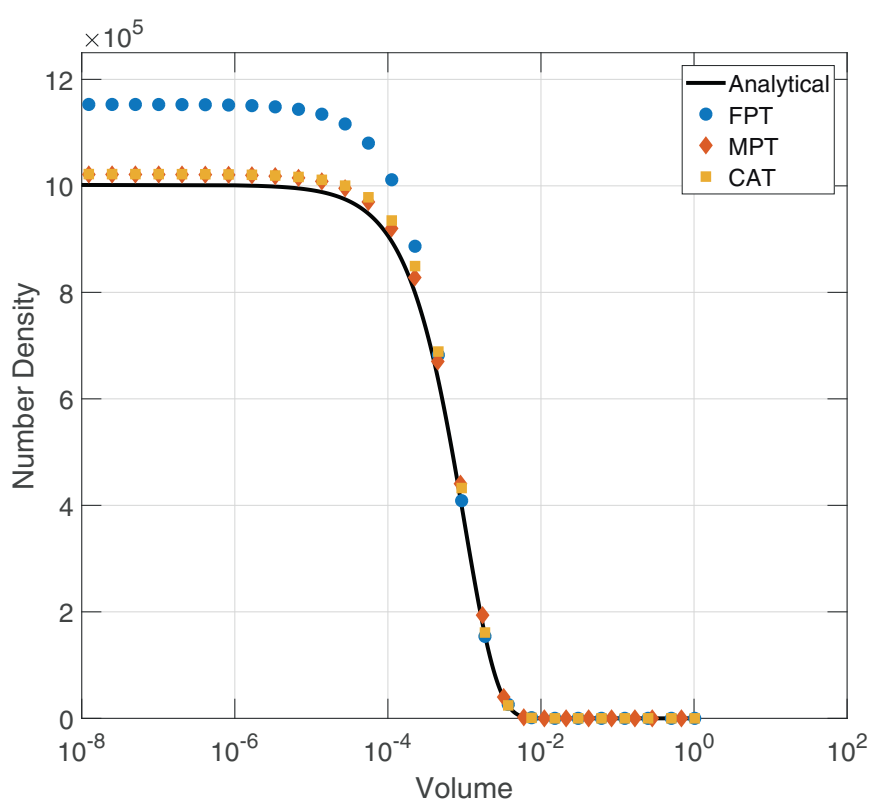

(a) 30 Discretizations

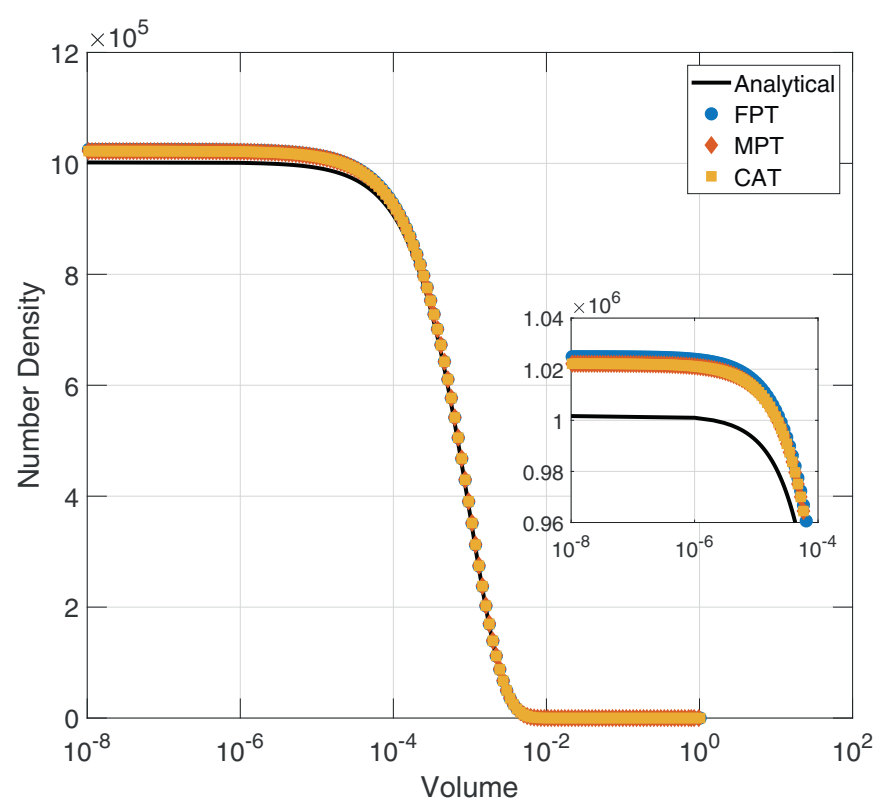

(b) 200 Discretizations

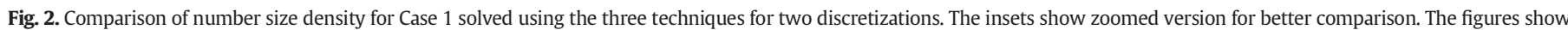
that, for all discretizations FPT overestimates the number density. With increasing discretizations, all the three methods converge closer to the analytical solution.

$\frac{d x_{i}}{d t}=-\frac{1}{N_{i}} \sum_{j \geq i} S\left(x_{j}\right) N_{j}\left[\beta_{i, j}^{(I I)}-x_{i} \beta_{i, j}^{(I)}\right]$

where,

$\beta_{i, j}^{(I)}=\int_{x_{i}}^{x_{i+1}} b\left(x, x_{j}\right) d x \quad \beta_{i, j}^{(I I)}=\int_{x_{i}}^{x_{i+1}} x b\left(x, x_{j}\right) d x$

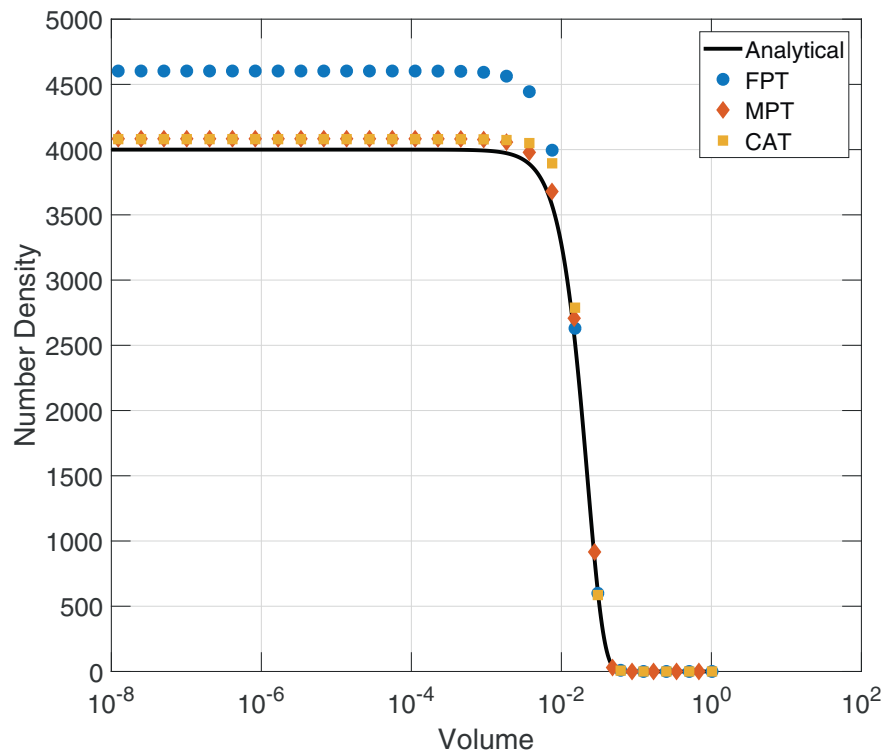

(a) 30 Discretizations
Eq. (7) describes the variation of the pivot location based on the determination of the average size of each bin when new particles are born in them.

\subsubsection{Cell average technique}

The cell average technique (CAT) was proposed by Kumar et al. [37] to solve a 1-dimensional PBM. The method distributes the total number of particles born in a cell to neighbouring nodes based on an averaging strategy described further in this section.

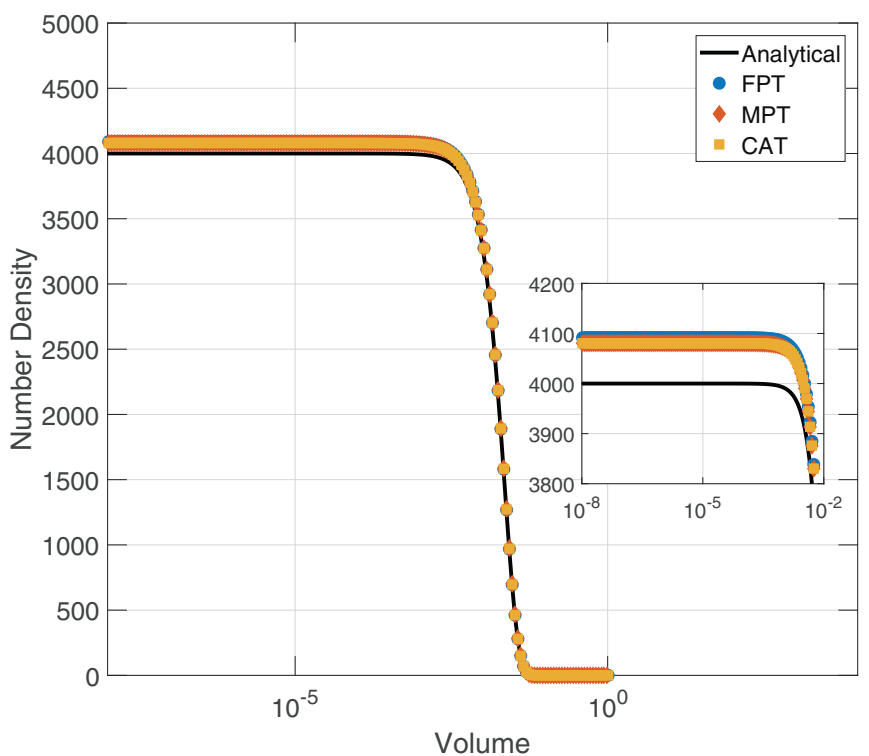

(b) 200 Discretizations

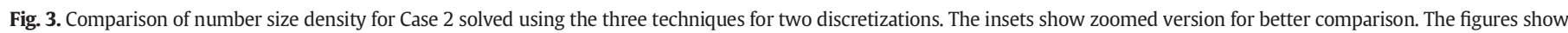
that, for all discretizations FPT overestimates the number density. With increasing discretizations, all the three methods converge closer to the analytical solution. 


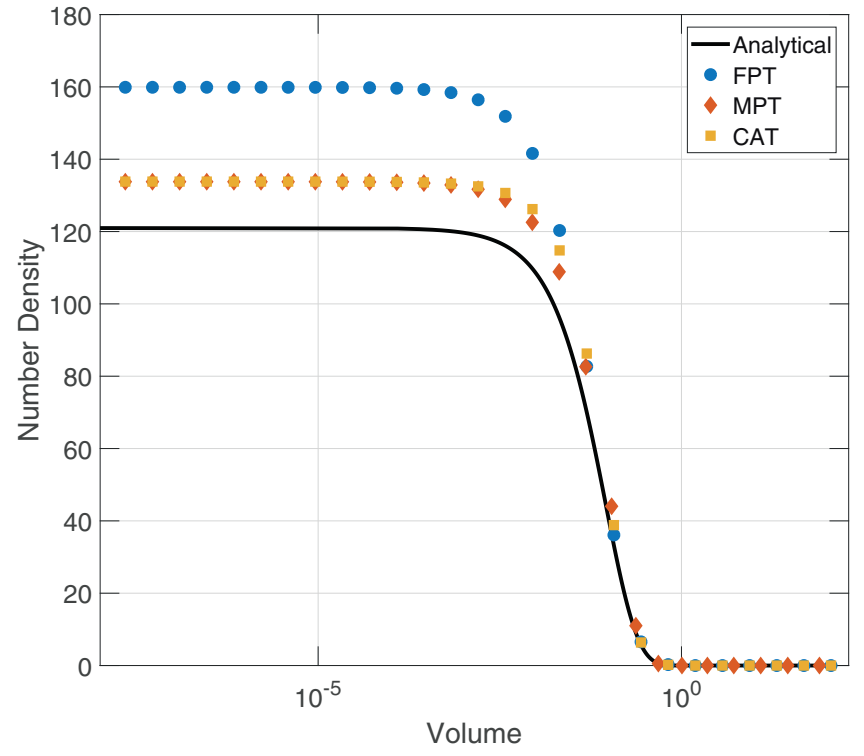

(a) 30 Discretizations

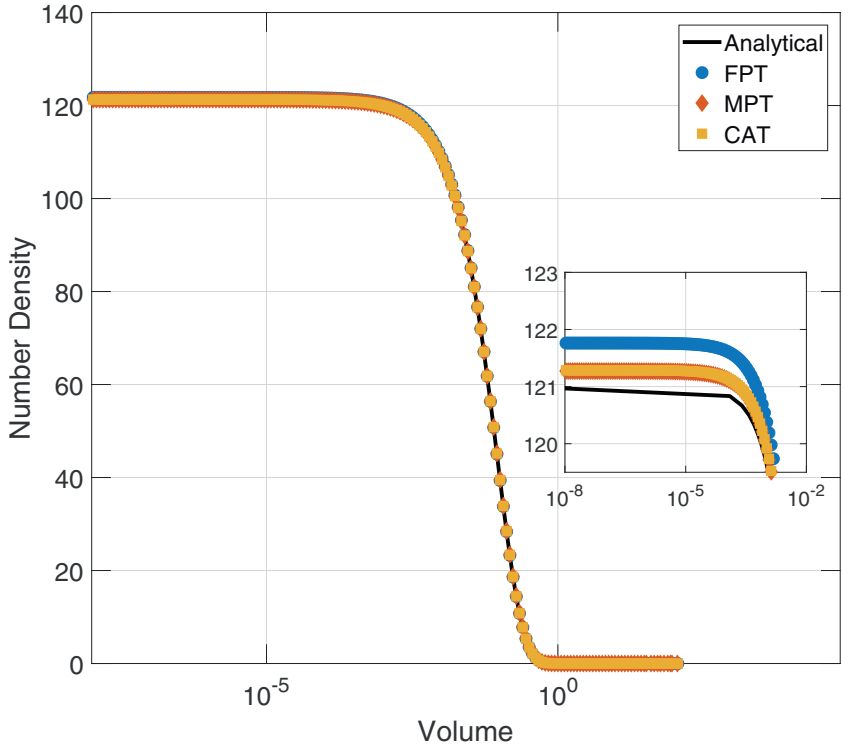

(b) 200 Discretizations

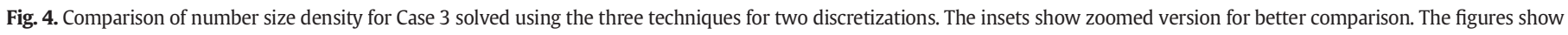
that, for all discretizations FPT overestimates the number density. With increasing discretizations, all the three methods converge closer to the analytical solution.

The PBM for breakage can be discretized to give the following form and

$\frac{d N_{i}}{d t}=\mathscr{B}_{i}^{\mathrm{CA}}-\mathcal{D}_{i}^{\mathrm{CA}}$

$\mathcal{D}_{i}^{\mathrm{CA}}=S_{i} N_{i}$

The terms $\mathscr{B}_{i}{ }^{\mathrm{CA}}$ and $\mathcal{D}_{i}^{\mathrm{CA}}$ represent the birth and death rates as calculated by the cell average strategy. For processes involving only breakage, these terms are given as

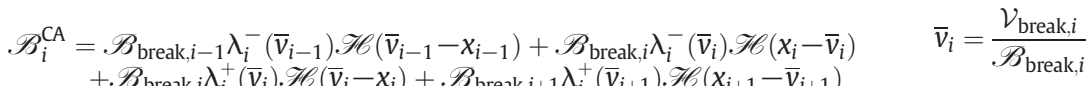

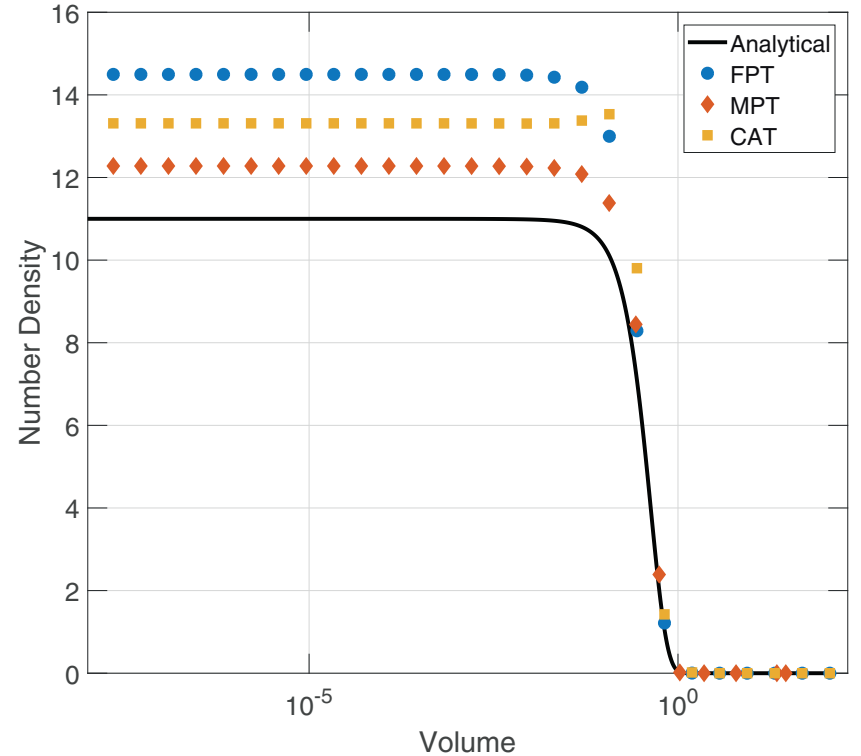

(a) 30 Discretizations

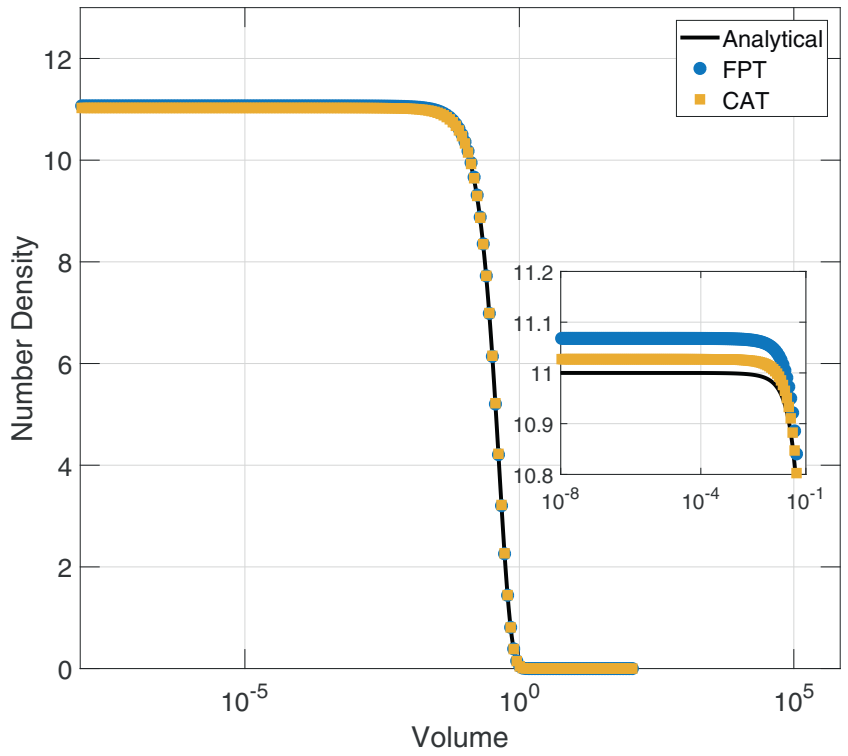

(b) 200 Discretizations

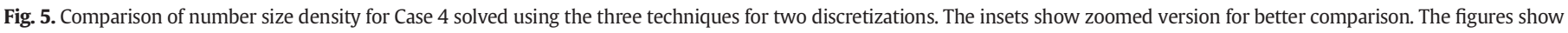

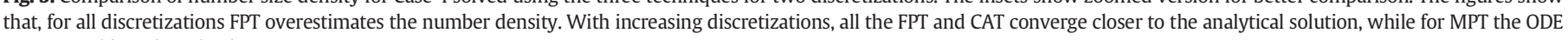
system could not be solved. 
The total breakage in a cell is calculated as

$\mathscr{B}_{\text {break }, i}=\sum_{k \geq i} N_{k} S_{k} \int_{x_{i-1 / 2}}^{p_{i}^{k}} b\left(x, x_{k}\right) d x$

where the integration limit is defined as

$p_{i}^{k}= \begin{cases}x_{i} & \text { if } k=i \\ x_{i+1 / 2} & \text { otherwise }\end{cases}$

The discrete volume flux is computed as

$\mathcal{V}_{\text {break }, i}=\sum_{k \geq i} N_{k} S_{k} \int_{x_{i-1 / 2}}^{p_{i}^{k}} x b\left(x, x_{k}\right) d x$

The discontinuous Heaviside function is described by

$\mathscr{H}(x)=\left\{\begin{array}{lll}1 & \text { if } & x>0 \\ \frac{1}{2} & \text { if } & x=0 \\ 0 & \text { if } & x<0\end{array}\right.$

The $\lambda$ function is given by

$\lambda_{i}^{ \pm}(x)=\frac{x-x_{i \pm 1}}{x_{i}-x_{ \pm 1}}$

The set of ordinary differential equations obtained by the methods described above can be solved using any ODE solver to obtain the total number of particles in each cell. However, standard ODE solvers might lead to negative number densities. So, the tolerances of the integrators must be checked or positivity should be forced.

\subsection{Case studies with analytical solution}

As mentioned previously, analytical solutions of pure breakage processes described by Eq. (1) exist for a few limited cases. In these cases, Eq. (1) is solved by assuming simple forms of selection rate and initial particle number density. For the comparison of solution strategies, four test cases are considered. The analytical solutions to these problems, provided by Ziff and McGrady [41] are as follows

1. Case 1 . The first case considers linear breakage function and a monodispersed feed of size $L$. $t$ represents the time at which the solution is obtained. The solution is given by

$S(x)=x, \quad b(x, y)=\frac{2}{y}$

$n_{0}=\delta(x-L)$

$n(t)=\exp (-t x)\left(\delta(x-L)+\left[2 t+t^{2}(L-x)\right] \theta(L-x)\right)$

Table 2

Parameters and operating parameters for the cone mill adapted from [8].

\begin{tabular}{ll}
\hline Parameter & Value \\
\hline Inlet mass flow, $\dot{m}_{\text {in }}$ & $7.4 \times 10-3 \mathrm{~kg} / \mathrm{s}$ \\
Particle density, $\rho$ & $740 \mathrm{~kg} / \mathrm{m}^{3}$ \\
Inlet distribution median, $\mu_{\text {in }}$ & $4.81 \times 10^{-5} \mathrm{~m}^{3}$ \\
Inlet distribution deviation, $\sigma_{\text {in }}$ & 0.25 \\
Selection reference volume, $x_{\text {ref }}$ & $2.23 \times 10^{-9} \mathrm{~m}^{3}$ \\
Critical screen size parameter, $\delta$ & 0.44 \\
Selection function parameter, $\alpha$ & $8.82 \times 10^{-6}$ \\
Selection function parameter, $\gamma$ & 0.34 \\
Breakage distribution function parameter, $n$ & $2.68 \times 10^{5}$ \\
Breakage distribution parameter, $\sigma$ & 2.10 \\
Impeller speed, $v_{\text {imp }}$ & $4923 \mathrm{RPM}$ \\
Screen aperture, $d_{\text {screen }}$ & $1.575 \times 10^{-3} \mathrm{~m}$ \\
\hline
\end{tabular}

where $\theta(x-L)$ is defined as

$\theta(x-L)= \begin{cases}1, & \text { if } x<L \\ 0, & \text { else }\end{cases}$

2. Case 2 . The second case considers quadratic breakage function and a monodispersed feed of size $L$. The solution is given by

$$
\begin{aligned}
& S(x)=x^{2}, \quad b(x, y)=\frac{2}{y} \\
& n 0=\delta(x-L) \\
& n(t)=\exp \left(-t x^{2}\right)[\delta(x-L)+2 t L \theta(L-x)]
\end{aligned}
$$

where $\theta(x-L)$ is defined in Case 1 .

3. Case 3. The third case considers linear breakage function and a feed size described by $n_{0}$. The solution is given by

$$
\begin{aligned}
& S(x)=x, \quad b(x, y)=\frac{2}{y} \\
& n_{0}=\exp (-x) \\
& n(t)=\exp (-x(1+t))(1+t)^{2}
\end{aligned}
$$

4. Case 4. The fourth case considers quadratic breakage function and a feed size described by $n_{0}$. The solution is given by

$$
\begin{aligned}
& S(x)=x^{2}, \quad b(x, y)=\frac{2}{y} \\
& n_{0}=\exp (-x) \\
& n(t)=\exp \left(-t x^{2}-x\right)[1+2 t(1+x)]
\end{aligned}
$$

\subsection{Cone mill model}

The model of Barrasso et al. [8] describes the evolution of the number of particles over time with respect to the particle size represented by its volume.

$\frac{\partial n(x, t)}{\partial t}=\dot{n}_{\text {in }}(x, t)-\dot{n}_{\text {out }}(x, t)-\mathcal{D}(x, t)+\mathscr{B}(x, t)$

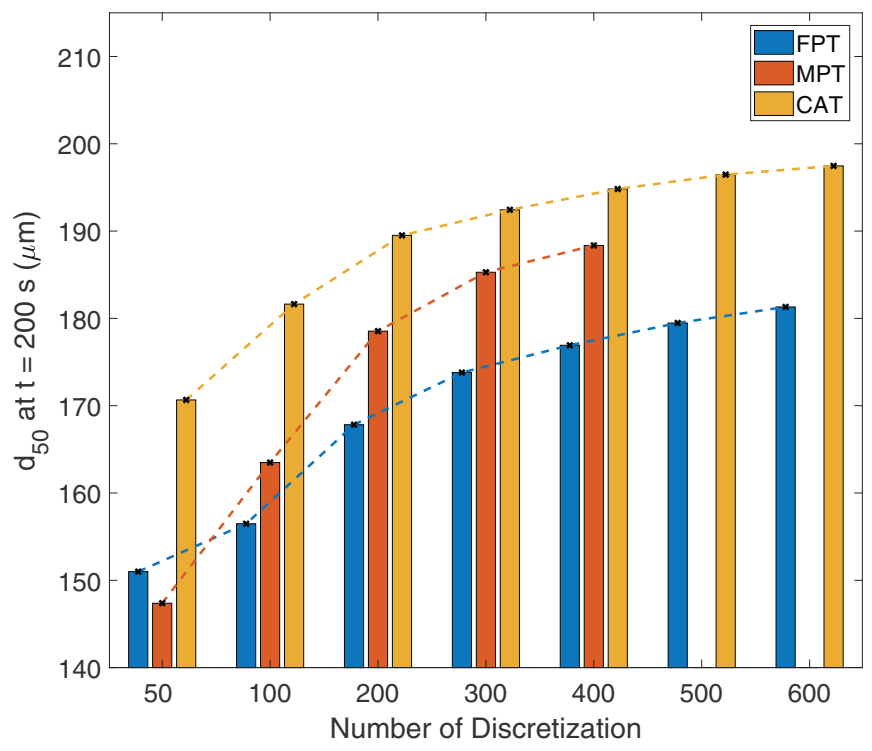

Fig. 6. The $d_{50}(\mu m)$ at $t=200$ s obtained by solving the PBM by FPT, MPT and CAT for different discretizations. It can be observed that with increasing discretization, the value of $d_{50}$ starts to converge. However, the convergence is not yet achieved for the discretizations considered. 
Here, $n(x, t)$ is the number of particles of volume $x$ in the mill at any time $t$. This model is a simple extension of the batch breakage equation described in Eq. (1) to a continuous system by including the feed inlet $\left(\dot{n}_{\text {in }}(x, t)\right)$ and the product outlet $\left(\dot{n}_{\text {out }}(x, t)\right)$.

$\dot{n}_{i n}(x, t)$, the number of particles being fed to the mill, is calculated from the mass flow rate $\left(\dot{m}_{i n}\right)$ as

$\dot{n}_{\text {in }}(x, t)=\frac{f_{\text {in }}(x)}{\sum f_{\text {in }}(x)} \frac{\dot{m}_{\text {in }}}{\rho x}$

with $\rho$ being the density of the particles, and $f_{\text {in }}(x)$ being the volumeweighted distribution of the feed particles. $\dot{n}_{\text {out }}(x, t)$ is the outlet flow based on the following screen classification model.

$\dot{n}_{\text {out }}(x, t)=\left[\dot{n}_{\text {in }}(x, t)-\mathcal{D}(x, t)+\mathscr{B}(x, t)\right]\left[1-f_{d}(x)\right]$

$f_{d}(x)= \begin{cases}0 & \text {,if } d(x) \leq(1-\delta) d_{\text {screen }} \\ \frac{d(x)-(1-\delta) d_{\text {screen }}}{\delta d_{\text {screen }}} & , \text { if }(1-\delta) d_{\text {screen }}<d(x) \leq d_{\text {screen }} \\ 1 & \text {,if } d(x)>d_{\text {screen }}\end{cases}$

where $d(x)$ is the particle diameter associated with volume $x . d_{\text {screen }}$ is the screen opening, and $\delta$ is a parameter which determines the critical diameter. A particle with diameter larger than the screen opening will be retained in the mill, whereas a particle smaller than the critical diameter will exit the mill. The screen is non-ideal

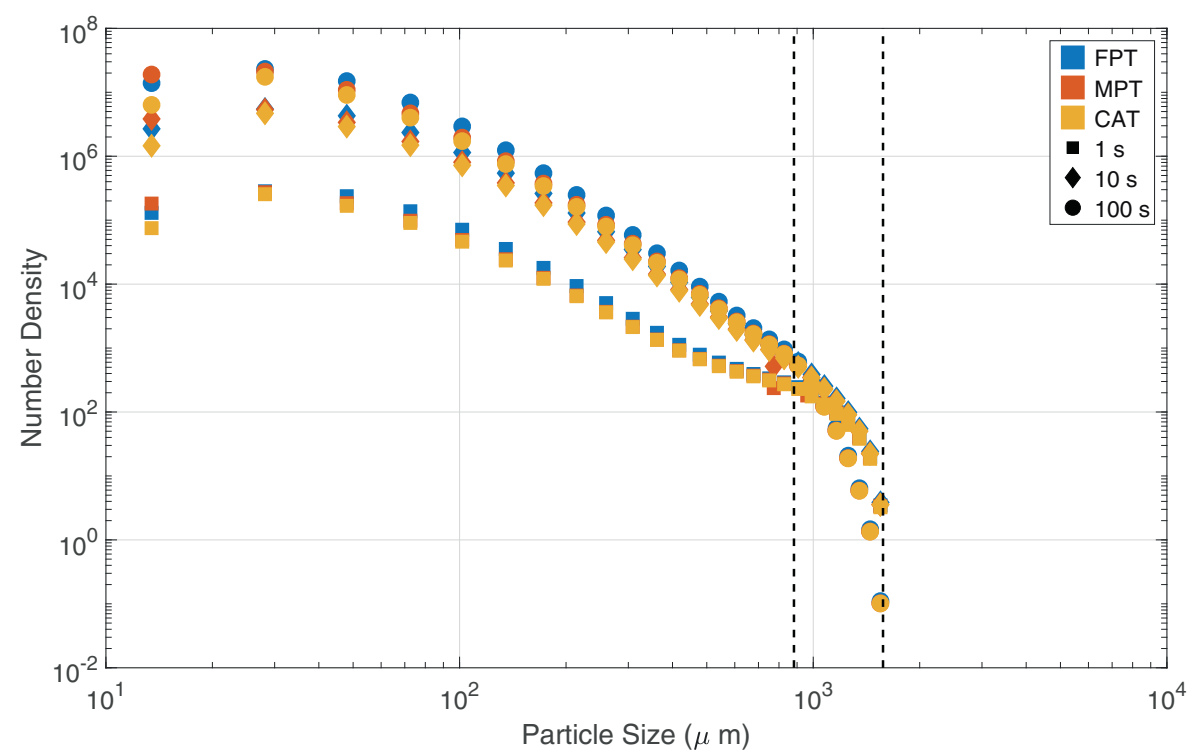

(a) 200 Discretization

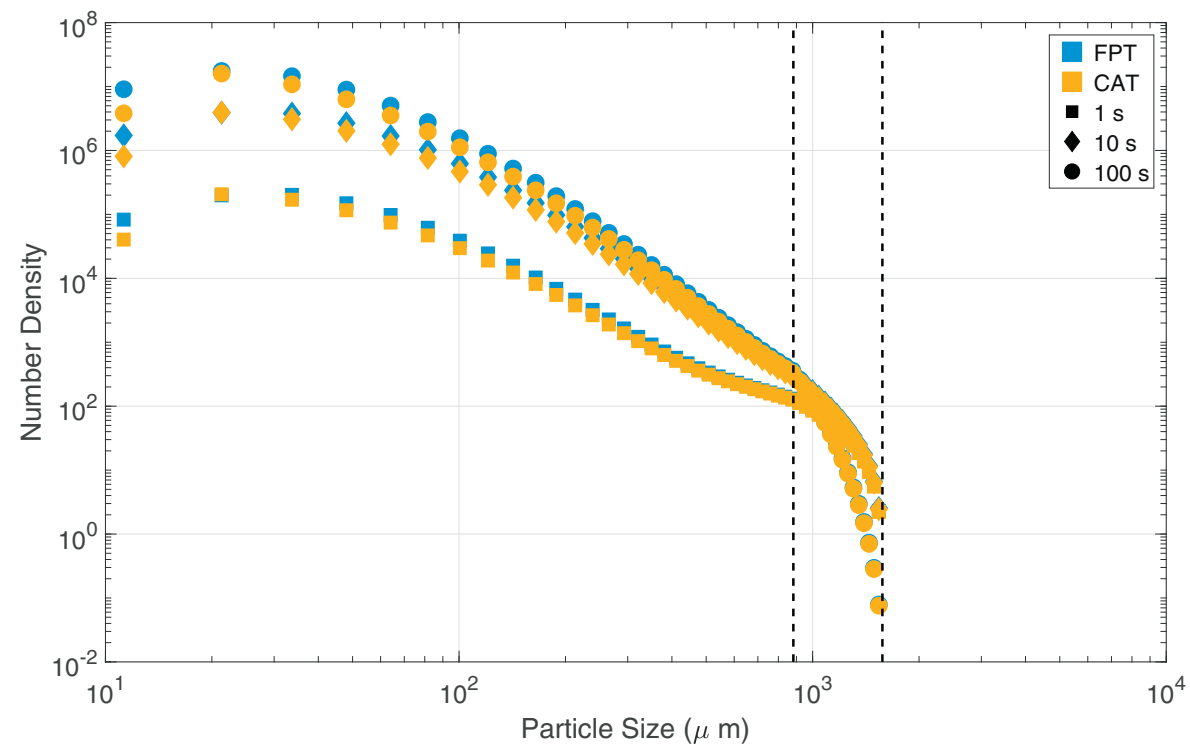

(b) 500 Discretization

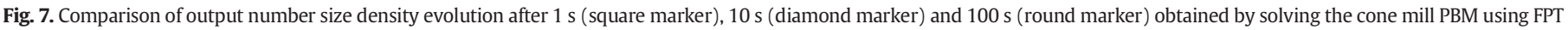

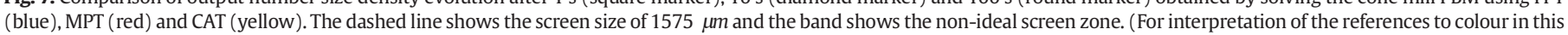
figure legend, the reader is referred to the web version of this article.) 
for particle diameters between the screen opening and the critical diameter.

The birth and death of the particles is represented by $\mathscr{R}(x, t)$ and $\mathcal{D}(x, t)$

$\mathscr{B}(x, t)=\int_{x}^{\infty} b(x, y) S(y) n(y, t) d y$

$\mathcal{D}(x, t)=S(x) n(x, t)$

The breakage rate depends on the particle and process parameters and is represented as with $\alpha$ and $\gamma$ as model parameters that need to be tuned, and $v_{\text {imp }}$ is the impeller speed of the cone mill shaft. The breakage distribution function is chosen to be a log-normal function

$b(x, y)=\frac{C(y)}{x \sigma} \exp \left[-\frac{\left(\log x-\log \frac{y}{n}\right)^{2}}{2 \sigma^{2}}\right]$

The volume of the parent particle is represented by $y$, and $C(y)$ is chosen to fulfil the mass conservation constraint

$\int_{0}^{y} x b(x, y) d x=y$

$S(x)=\alpha v_{\text {imp }}\left(\frac{x}{x_{\text {ref }}}\right)^{\gamma}$

The unknown tuneable parameters in the above model are estimated from experimental data. The estimation of these parameters is

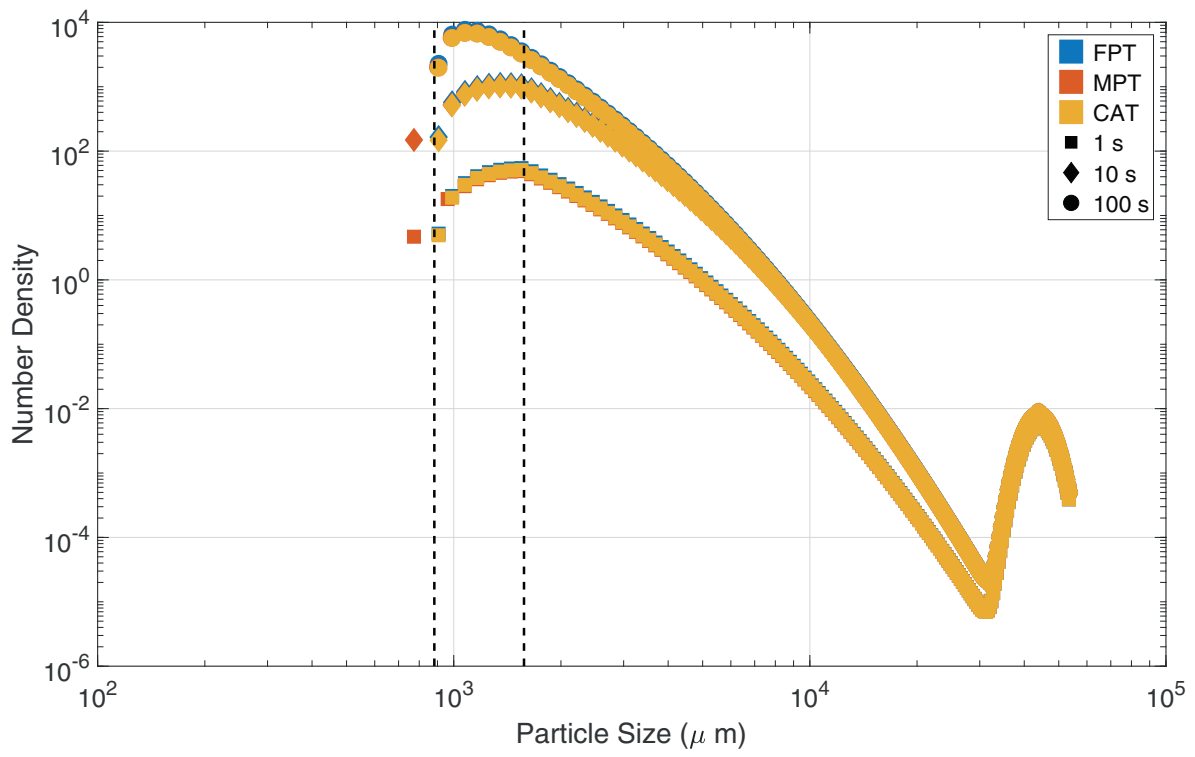

(a) 200 Discretization

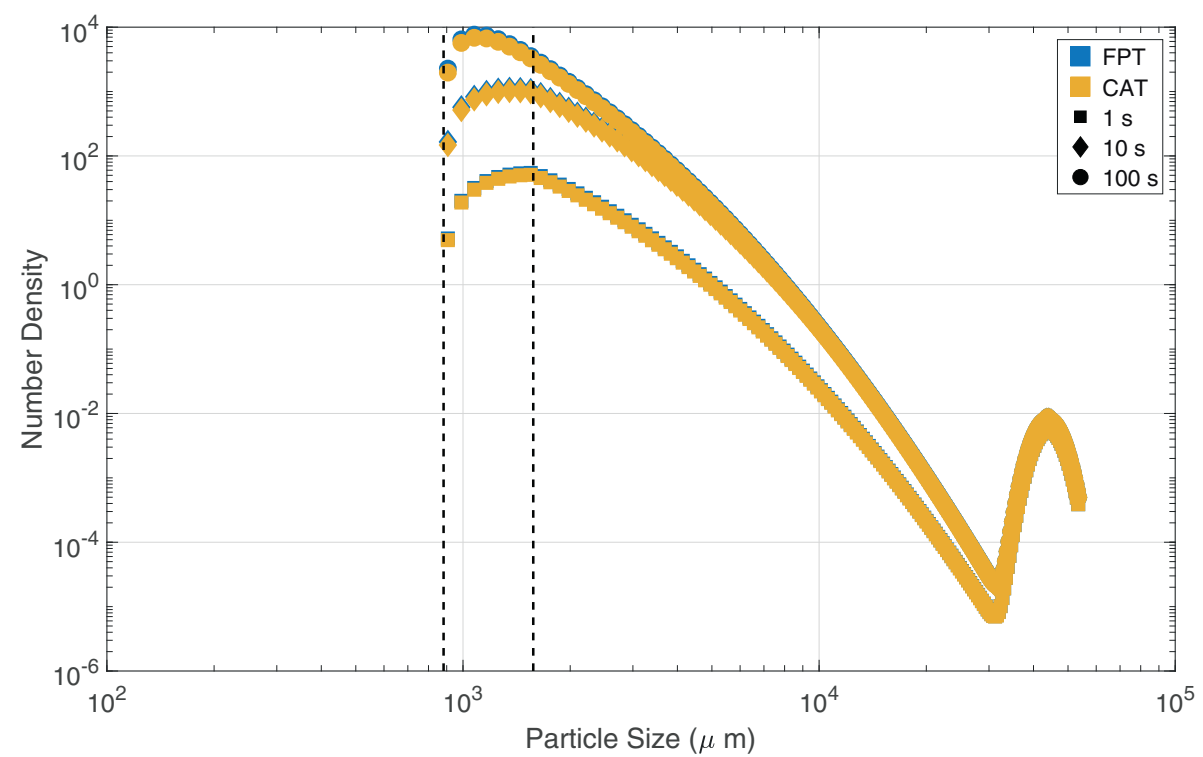

(b) 500 Discretization

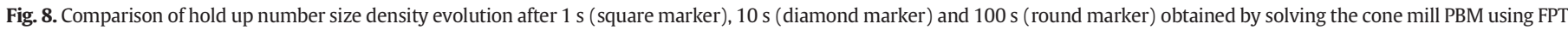

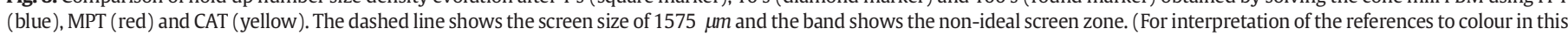
figure legend, the reader is referred to the web version of this article.) 
not the focus of this work, and the parameters obtained in [8] will be used. However, accuracy of the model parameters is an important factor while considering the use of the model for prediction. Any uncertainty in the parameter estimate will be propagated to the model prediction. A study on such uncertainty propagation is presented in [42].

\section{Numerical results}

\subsection{Test cases with analytical solutions}

In this section, the performance of the FPT, MPT and CAT will be evaluated and compared for the four test cases described in Section 2.2. For all cases, the MATLAB integrator ode45 is used with default integration tolerances. The average number densities are compared at the end of each milling batch. The batch times for each case are mentioned in Table 1.
Fig. 1 depicts the mean and the maximum L1 error of the three methods over 5 discretizations. It can be observed that the error in all cases starts to converge to a constant value, i.e., after certain grid density, further refining of the grid will only lead to increased computational cost and not increased accuracy. However, attainment of this grid independent solution is case and method dependant. For the fourth case, only three discretizations were studied for the moving pivot case. At discretizations of 150 and 200, the system becomes extremely stiff and the standard integrator used fails. A rough indicator for the stiffness of the system is the condition number of the Jacobian matrix of the ODE system. For this case, the condition number obtained was infinity (Inf). The Inf arises from the fact that the Jacobian was approximated by a finite difference approximation. True calculation of Jacobian will lead to a non-infinite, but extremely large condition number.

In Fig. 2, the number density obtained analytically is compared with the number density obtained numerically by the three methods with four different discretizations. The initial condition and the simulation

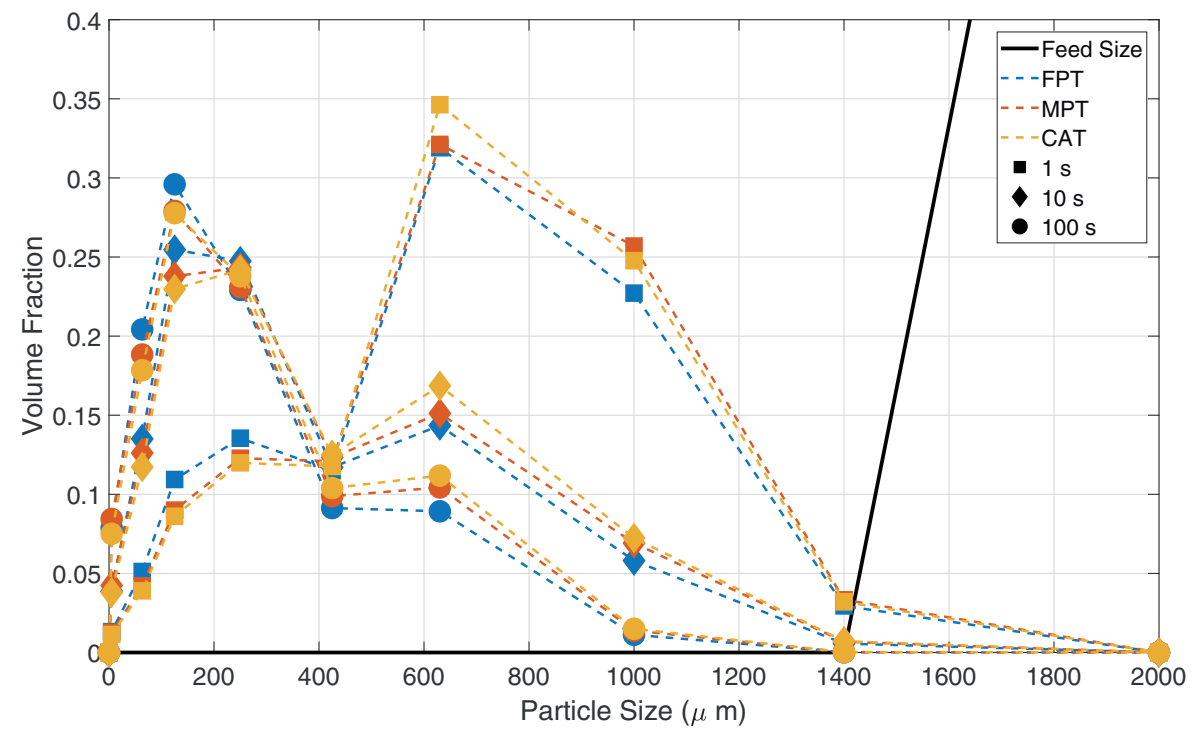

(a) 200 Discretization

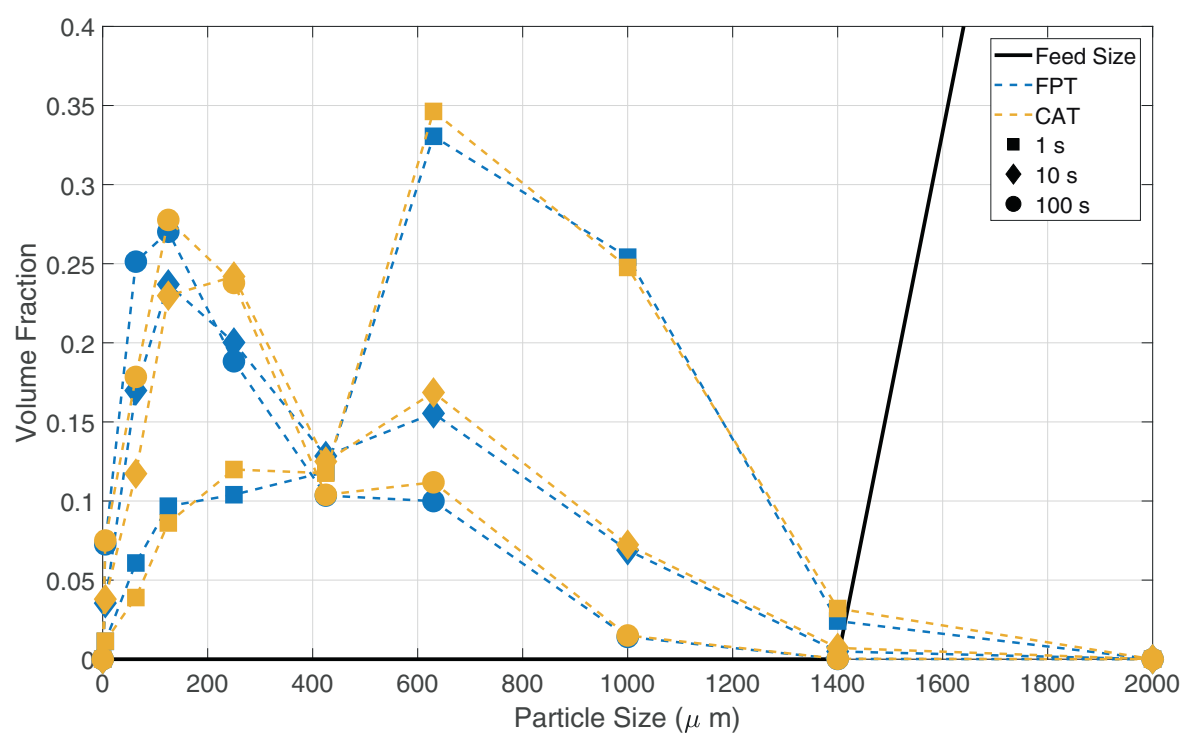

(b) 500 Discretization

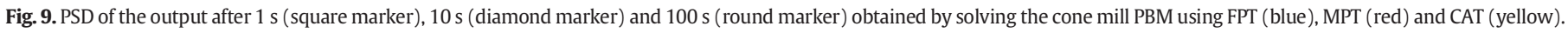

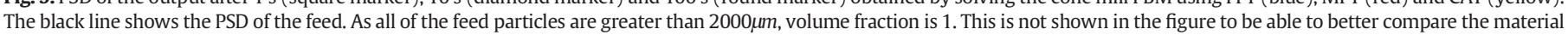
after breakage. (For interpretation of the references to colour in this figure legend, the reader is referred to the web version of this article.) 
conditions used are reported in Section 2.2 and Table 1 respectively. On the semi-logarithmic scale of Fig. 2a, it can be seen that the FPT overestimates the number density. Whereas both the CAT and MPT approximate the analytical solution well. Fig. $2 \mathrm{~b}$ shows that using a finer grid improves the performance of all the three methods. Although the improvement in FPT performance is significant, the method still over predicts the number density at the finer range. Moreover, after 90 discretizations, the performance of all the methods stabilizes and finer grids do not lead to a better solution. As expected, the computational time also increases with the fineness of the grid. The computational times for all cases are reported in Table 1.

For the second case, the selection function $S(x)$ is quadratic. It can be seen from Fig. 3 that the FPT again overpredicts the number density, whereas MPT and CAT are in good agreement with the analytical solution for all discretizations. Increasing the number of grid points again causes an improvement in all three methods, with the improvement in FPT being the most significant. However, with increasing discretizations the three methods converge towards a constant error.

In the third case, the initial particle distribution is an exponential function of the particle size, with a linear selection function. It can be noticed from Fig. 4a, that all three methods fail to converge to the analytical solution. The FPT performs the worst, whereas MPT performs slightly better than CAT. A finer grid has a significantly better performance for all three methods. A discretization with 200 points (Fig. 3b) gives a good approximation of the analytical solution. However, as can be observed from Fig. 1c, a grid independent solution is already obtained from 90 discretization points. Further increase in the fineness of the grid does not lead to any significant increase in the accuracy.

The fourth case has an exponential initial condition and quadratic selection function. Fig. 5a shows that MPT clearly performs the best among the three methods. The performance of all the methods increases when the grid is refined. However, when the grid is refined to 150 grid points or more, the numerical system arising from MPT cannot be solved using standard MATLAB solvers. Even with the stiff MATLAB sovler ode15s, the integration tolerances had to be reduced to be able to get a solution of the system. However, with reduced tolerances the solution tends to have negative values for the number density. CAT performs better than FPT on all grids. As in the previous cases, the methods converge to a constant error with increasing grid refinement.

In general, it can be said that although MPT performs the best for all cases and discretization, the computational cost might not warrant the use of this technique. CAT is relatively fast and overcomes the drawback of FPT effectively.

\subsection{Cone mill}

In this section, the PBM for cone mill as described by Eqs. (18)-(26) is solved using the three methods. The MATLAB ode45 solver is used with strict tolerance to avoid any negative number density values. The relative tolerance is set to $10^{-14}$ and absolute tolerance to $10^{-17}$. The integrations required to evaluate the constant $C(y)$ in Eq. (25) are carried out using Gaussian quadrature method available in MATLAB integral routine. For the numerical integration, both the relative and absolute tolerances are set to $10^{-18}$. Unlike the previous case studies, the breakage distribution and selection rate functions are complex mathematical functions. Even analytical integration of Eq. (25) is not possible. This makes the analytical solution of the PBM impossible. The three methods are thus compared to each other using average number densities in the hold up and output, and sieved output in terms of volume fractions. The particle volume is the continuous internal state. The use of MPT for continuous system requires an adaptation to the Eq. (7), to consider the effect of inlet feed on the pivot location [43]. The simulation conditions and simulation parameters are given in Table 2.

Fig. 6 shows the $d_{50}$ values obtained by solving the PBM using the three methods at various discretizations. The $d_{50}$ is the median particle size of the distribution calculated on a volume basis. It can be noticed that with increasing discretizations the value of $d_{50}$ starts to converge to a constant value. However, this constant value is not obtained for the discretizations studied here. The FPT consistently provides a lower $d_{50}$ value than CAT and MPT (except at 50 discretizations). It must be noticed that even at 600 discretizations, the difference between FPT and CAT is significant. For batch systems not involving inlet and outlet terms, both CAT and FPT are known to be second order convergent on smooth grids $[44,45]$. However, the addition of inlet and outlet terms seems to affect the convergence rate and convergence is not achieved up till 600 discretizations. According to [44,45], the experimental error of convergence can be calculated as

$E O C=\frac{\ln \left[\left\|N_{I}-N_{2 I}\right\| /\left\|N_{2 I}-N_{4 I}\right\|\right]}{\ln (2)}$

The EOC of FPT, MPT and CAT for 400 discretizations is $0.32,0.61$ and 0.56 respectively. This low rate of convergence can explain why convergence is not achieved up to 600 discretizations.

Figs. 7 and 8 depict the evolution of the output particle size, and the hold up particle size at three times: $1 \mathrm{~s}, 10 \mathrm{~s}, 100 \mathrm{~s}$. For the two discretizations considered here, there is a difference between the number densities obtained by the three methods at the finer end of the grid. Just as FPT has consistently overestimated the amount of fines for the case studies, FPT also seems to have higher amount of fines, which are consequently removed from the mill (Fig. 7). Even MPT calculates a higher amount of fines than CAT. This shows why the $d_{50}$ values are lower for both FPT and MPT. The difference between the number densities obtained by FPT and CAT is reduced when the grid is refined. The slight bump in the distribution in Fig. 8 is the particle size of the feed.

In an experimental system, the output PSD is usually measured by sieving the product from the cone mill. To simulate these conditions, the number density obtained is converted to volume fraction by assumption of spherical particles, and then reassigned to a coarser grid representing the sieve sizes. Fig. 9 shows the particle size after simulating such a sieve analysis into sieves with openings of $2000 \mu \mathrm{m}, 1400 \mu \mathrm{m}$, $1000 \mu \mathrm{m}, 630 \mu \mathrm{m}, 425 \mu \mathrm{m}, 125 \mu \mathrm{m}, 63 \mu \mathrm{m}$ and $5 \mu \mathrm{m}$. The particle sizes are compared at three different times: after $1 \mathrm{~s}, 10 \mathrm{~s}$ and $100 \mathrm{~s}$. It can be noticed that at this sieve resolution, the difference in the particle size distribution obtained by the different methods is not much. FPT again shows slightly higher amount of fines than the other two methods, but this is not as significant as in the average number density. Experimentally, sieving is normally prone to errors. The errors can arise due to the sieves themselves, the method of sieving or the sample. As such, the difference in the PSD observed in Fig. 9 can be considered within the error of sieving.

In most cases, the parameters of the model need to be estimated using the experimental sieving data. Based on Fig. 9, it can be noted that choice of discretization method will not significantly affect parameter estimation. However, as is clear from Fig. 6, the choice of discretization affects both the median size and the average number density. So, the choice of the discretization method must be motivated by the application of the model.

\section{Conclusions}

The numerical solutions to PBMs obtained by three sectional methods have been compared for different cases. At very coarse grids MPT provides the best approximation of the analytical solution for the cases considered in Section 2.2. However, computational time required for MPT is much higher than the time required for FPT and CAT. As the grid becomes finer, this cost increases even further. It should be noted, that all the three methods exhibit a certain amount of bias even at high grid densities. All the three methods become grid independent as the grids become denser with the biases converging to constant values. However, at what grid resolution such a grid independent solution is 
achieved depends on the case and the method. At a high grid density, CAT outperforms the other methods by a slight margin.

The results for the cone mill follow a similar trend as those for the analytical cases. With increasing grid density, the $d_{50}$ values start converging to a constant value. However, a grid independent solution is not yet obtained for the grids considered here. FPT and MPT consistently provide lower $d_{50}$ values than CAT. This is due to the higher amount of fines computed by FPT and MPT. Based on the results of Section 3.1 we can consider the higher number density at finer sizes an artefact of the FPT method. In MPT, the particle size pivot changes position based on the number densities. Unlike the cases in Section 2.2, the breakage rate in the cone mill is significantly higher. This can cause the pivot to move rapidly towards finer range causing the depression in $d_{50}$ values. In any case, the computational time required for MPT for the cone mill PBM is orders of magnitude larger than the computational times required for CAT and FPT making it an unattractive method for breakage PBM. Between CAT and FPT, we can conclude that for coarser grids CAT provides a more accurate approximation of the solution. This however comes at the cost of slightly higher computational cost.

\section{Acknowledgements}

This work was supported by Project G0863.18 of the Fund for Scientific Research-Flanders. SB holds an IWT-Baekeland grant [IWT150715].

\section{References}

[1] G.G. Liversidge, K.C. Cundy, Particle size reduction for improvement of oral bioavailability of hydrophobic drugs: I. Absolute oral bioavailability of nanocrystalline danazol in beagle dogs, Int. J. Pharm. 125 (1) (1995) 91-97.

[2] M. Vogt, K. Kunath, J.B. Dressman, Dissolution enhancement of fenofibrate by micronization, cogrinding and spray-drying: Comparison with commercial preparations, Eur. J. Pharm. Biopharm. 68 (2) (2008) 283-288.

[3] J.E. Byers, G.E. Peck, The effect of mill variables on a granulation milling process, Drug Dev. Ind. Pharm. 16 (11) (1990) 1761-1779.

[4] J.J. Verheezen, K. van der Voort Maarschalk, F. Faassen, H. Vromans, Milling of agglomerates in an impact mill, Int. J. Pharm. 278 (1) (2004) 165-172.

[5] J.J. Motzi, N.R. Anderson, The quantitative evaluation of a granulation milling process II. Effect of ouput screen size, mill speed and impeller shape, Drug Dev. Ind. Pharm. 10 (5) (1984) 713-728.

[6] A. Vanarase, R. Aslam, S. Oka, F. Muzzio, Effects of mill design and process parameters in milling dry extrudates, Powder Technol. 278 (2015) 84-93.

[7] L.R. Schenck, R.V. Plank, Impact milling of pharmaceutical agglomerates in the wet and dry states, Int. J. Pharm. 348 (1) (2008) 18-26.

[8] D. Barrasso, S. Oka, A. Muliadi, J.D. Litster, C. Wassgren, R. Ramachandran, Population balance model validation and prediction of CQAs for continuous milling processes: Towards QbD in pharmaceutical drug product manufacturing, J. Pharm. Innov. 8 (3) (2013) 147-162.

[9] M. Ghadiri, Z. Zhang, Impact attrition of particulate solids. Part 1: a theoretical model of chipping Chem. Eng. Sci. 57 (17) (2002) 3659-3669.

[10] P. Cundall, O. Strack, Discrete numerical model for granular assemblies, Geotechnique 29 (1) (1979) 47-65

[11] J. Zhang, Z. Hu, W. Ge, Y. Zhang, T. Li, J. Li, Application of the discrete approach to the simulation of size segregation in granular chute flow, Ind. Eng. Chem. Res. 43 (18) (2004) 5521-5528.

[12] R. Yang, A. Yu, L. McElroy, J. Bao, Numerical simulation of particle dynamics in different flow regimes in a rotating drum, Powder Technol. 188 (2) (2008) 170-177.

[13] D. Suzzi, G. Toschkoff, S. Radl, D. Machold, S.D. Fraser, B.J. Glasser, J.G. Khinast, DEM simulation of continuous tablet coating: Effects of tablet shape and fill level on intertablet coating variability, Chem. Eng. Sci. 69 (1) (2012) 107-121.

[14] N. Weerasekara, M. Powell, P. Cleary, L. Tavares, M. Evertsson, R. Morrison, J. Quist, R. Carvalho, The contribution of DEM to the science of comminution, Powder Technol. 248 (2013) 3-24 (discrete Element Modelling).

[15] X. Deng, J. Scicolone, X. Han, R.N. Dave, Discrete element method simulation of a conical screen mill: a continuous dry coating device, Chem. Eng. Sci. 125 (2015) 58-74 (pharmaceutical Particles and Processing).
[16] T. Brosh, H. Kalman, A. Levy, DEM simulation of particle attrition in dilute-phase pneumatic conveying, Granul. Matter 13 (2) (2011) 175-181.

[17] E.G. Ardi, K. Dong, A. Yu, R. Yang, A combined experimental and DEM approach to determine the breakage of particles in an impact mill, Powder Technol. 318 (2017) 543-548.

[18] S. Naik, B. Chaudhuri, Quantifying dry milling in pharmaceutical processing: a review on experimental and modeling approaches, J. Pharm. Sci. 104 (8) (2015) 2401-2413.

[19] M. Capece, E. Bilgili, R. Dave, Insight into first-order breakage kinetics using a particle-scale breakage rate constant, Chem. Eng. Sci. 117 (2014) 318-330.

[20] L. Vogel, W. Peukert, Breakage behaviour of different materials construction of a mastercurve for the breakage probability, Powder Technol. 129 (1) (2003) 101-110.

[21] M. Capece, R. Dave, E. Bilgili, A pseudo-coupled DEM nonlinear PBM approach for simulating the evolution of particle size during dry milling, Powder Technol. 323 (2018) 374-384.

[22] N. Metta, M. Ierapetritou, R. Ramachandran, A multiscale DEM - PBM approach for a continuous comilling process using a mechanistically developed breakage kernel, Chem. Eng. Sci. 178 (2018) 211-221.

[23] S. Loreti, C.-Y. Wu, G. Reynolds, A. MirtiÄ, J. Seville, Dem-pbm modeling of impact dominated ribbon milling, AICHE J. 63 (9) (2017) 3692-3705.

[24] D. Ramkrishna, Population Balances, Elsevier Inc, 2000.

[25] D.L. Marchisio, R. Vigil, R.O. Fox, Quadrature method of moments for aggregation breakage processes, J. Colloid Interface Sci. 258 (2) (2003) 322-334.

[26] C. Yuan, F. Laurent, R. Fox, An extended quadrature method of moments for population balance equations, J. Aerosol Sci. 51 (2012) 1-23.

[27] M.M. Attarakih, C. Drumm, H.J. Bart, Solution of the population balance equation using the sectional quadrature method of moments (SQMOM), Chem. Eng. Sci. 64 (4) (2009) 742-752 3rd International Conference on Population Balance Modelling.

[28] A. Falola, A. Borissova, XZ. Wang Extended method of moment for general population balance models including size dependent growth rate, aggregation and breakage kernels, Comput. Chem. Eng. 56 (2013) 1-11.

[29] F.E. Kruis, A. Maisels, H. Fissan, Direct simulation Monte Carlo method for particle coagulation and aggregation, AICHE J. 46 (9) (2000) 1735-1742.

[30] W.I. Friesen, T. Dabros, Constant-number Monte Carlo simulation of aggregating and fragmenting particles, J. Chem. Phys. 119 (5) (2003) 2825-2839.

[31] M.J. Hounslow, R.L. Ryall, V.R. Marshall, A discretized population balance for nucleation, growth, and aggregation, AICHE J. 34 (11) (1988) 1821-1832.

[32] J.D. Lister, D.J. Smit, M.J. Hounslow, Adjustable discretized population balance for growth and aggregation, AICHE J. 41 (3) (1995) 591-603.

[33] P.J. Hill, K.M. Ng, New discretization procedure for the breakage equation, AICHE J. 41 (5) (1995) 1204-1216.

[34] S. Kumar, D. Ramkrishna, On the solution of population balance equations by discretization I. A fixed pivot technique, Chem. Eng. Sci. 51 (8) (1996) 1311-1332.

[35] S. Kumar, D. Ramkrishna, On the solution of population balance equations by discretization II. A moving pivot technique, Chem. Eng. Sci. 51 (8) (1996) 1333-1342.

[36] M. Vanni, Discretization procedure for the breakage equation, AICHE J. 45 (4) (1999) 916-919.

[37] J. Kumar, M. Peglow, G. Warnecke, S. Heinrich, L. Mörl, Improved accuracy and convergence of discretized population balance for aggregation: the cell average technique, Chem. Eng. Sci. 61 (10) (2006) 3327-3342.

[38] D. Bertin, I. Cotabarren, J. Piña, V. Bucala, Population balance discretization for growth, attrition, aggregation, breakage and nucleation, Comput. Chem. Eng. 84 (2016) 132-150.

[39] J. Kumar, G. Warnecke, M. Peglow, S. Heinrich, Comparison of numerical methods for solving population balance equations incorporating aggregation and breakage, Powder Technol. 189 (2) (2009) 218-229.

[40] M. Singh, D. Ghosh, J. Kumar, A comparative study of different discretizations for solving bivariate aggregation population balance equation, Appl. Math. Comput. 234 (2014) 434-451.

[41] R.M. Ziff, E.D. McGrady, The kinetics of cluster fragmentation and depolymerization, J. Phys. A Math. Gen. 18 (1985) 3027-3037.

[42] S. Bhonsale, D. Telen, B. Stokbroekx, J.Van Impe, An analysis of uncertainty propagation methods applied to breakage population balance, Processes 6 (255) (2018).

[43] M. Attarakih, H.J. Bart, N. Faqir, Optimal moving and fixed grids for the solution of discretized population balances in batch and continuous systems: droplet breakage, Chem. Eng. Sci. 58 (7) (2003) 1251-1269.

[44] J. Kumar, G. Warnecke, Convergence analysis of sectional methods for solving breakage population balance equations-I: the fixed pivot technique, Numer. Math. 111 (1) (2008) 81-108.

[45] J. Kumar, G. Warnecke, Convergence analysis of sectional methods for solving breakage population balance equations-II: the cell average technique, Numer. Math. 110 (4) (2008) 539-559. 